\title{
Síndrome metabólica e baixa estatura em adultos da região metropolitana de São Paulo (SP, Brasil)
}

\author{
Metabolic syndrome and short stature \\ in adults from the metropolitan area of São Paulo city (SP, Brazil)
}

Elaine Cristina Silva ${ }^{1}$

Ignez Salas Martins

Eutália Aparecida Cândido de Araújo ${ }^{1}$

${ }^{1}$ Departamento de Nutrição, Faculdade de Saúde Pública, Universidade de São Paulo. Av. Dr. Arnaldo 715. 01246-904 São Paulo SP ecsilva@usp.br

\begin{abstract}
The undernutrition has been appointed as a risk factor to cause chronic diseases in later life. The objective of this study was to investigate the prevalence of metabolic syndrome and its relationship to short stature, as evidence of stunting in early life in adults. It is a cross-sectional study of adult population (age 20 to 64 years old) living in the metropolitan area of São Paulo city (SP, Brazil). The sample comprehended a total of 287 individuals, 214 (74.6\%) were women and 73 (25.4\%) were men. The anthropometric, clinical and biochemical data were collected through the physical exam and the fast blood after 8 hours. The standard prevalence for sex and age to metabolic syndrome was $34.0 \%$ and the short stature was $29.0 \%$. In multiple logistic regression analyses the metabolic syndrome showed to be associated to short stature adjusted by sex, age, education, income, smoking, alcohol consumption, family history of coronary disease, hypertension, diabetes and body mass index. In this study was demonstrated association between metabolic syndrome and short stature in adults. Key words Short stature, Metabolic syndrome, Risk factor, Prevalence
\end{abstract}

Resumo A desnutrição pregressa, expressa pela baixa estatura, pode ser fator de risco para síndrome metabólica (SM). O objetivo deste estudo foi verificar a prevalência de SM e sua relação com a baixa estatura, possível resultante de desnutrição crônica na infância, em população adulta. Foi feito um estudo transversal em população adulta, com idades entre 20 e 64 anos, residente em município da região metropolitana da cidade de São Paulo (SP). A amostra foi composta por 287 indivíduos, sendo 214 (74,6\%) do sexo feminino e 75 (25,4\%) do sexo masculino. Foram obtidos dados antropométricos, por meio de exame físico, dados bioquímicos (glicemia, colesterol total e frações, triglicérides) pela coleta de sangue em jejum e dados clínicos. A prevalência padronizada por sexo e idade para a síndrome metabólica foi de 34,0\% e para a baixa estatura, 29,0\%. A análise por regressão logística múltipla demonstrou associação entre a baixa estatura e a $S M$, ajustada por sexo, idade, escolaridade, renda, tabagismo, etilismo, atividade física, antecedentes familiares de doenças coronarianas, hipertensão arterial, diabetes e indice de massa corporal. Neste estudo, encontrou-se associação entre SM e baixa estatura, sugerindo que a desnutrição pregressa seja fator de risco para essa morbidade. Palavras-chave Baixa estatura, Sindrome metabólica, Fatores de risco, Prevalência 


\section{Introdução}

Por um longo período, a desnutrição e a fome eram as maiores causas de morbimortalidade em muitos países em desenvolvimento. A partir do século XX, porém, a industrialização, a urbanização, o desenvolvimento econômico e tecnológico acarretaram mudanças na alimentação e no estilo de vida, aumentando dramaticamente a prevalência de obesidade no mundo ${ }^{1-4}$.

No Brasil, essas mudanças têm sido mais evidentes nos últimos trinta anos, caracterizando a transição nutricional, em que a desnutrição apresenta acentuado declínio e a obesidade emerge como grave problema de saúde pública ${ }^{5,6}$. Entretanto, tem-se observado que tanto a desnutrição quanto a obesidade ainda coexistem com altas prevalências em populações de baixa renda ${ }^{7-9}$.

Estudos em animais têm demonstrado respostas adaptativas do organismo, mediante condições nutricionais específicas, com efeitos persistentes no decorrer da vida ${ }^{10-12}$. Segundo Barker $^{11}$, breves períodos de desnutrição podem reduzir o número de células em órgãos específicos, um dos mecanismos pelos quais a desnutrição poderia mudar a "memória" do corpo, desencadeando mudanças na distribuição de tipos celulares, na atividade metabólica e nas estruturas dos órgãos, levando desta forma ao desenvolvimento de doenças na vida adulta. Alguns autores têm usado o termo "programação" para referir-se a esses efeitos ${ }^{10-12}$.

Em humanos, os estudos epidemiológicos têm demonstrado que a desnutrição na infância aumenta o risco de obesidade no futuro ${ }^{13-20}$. Um dos estudos pioneiros foi apresentado por Ravelli et $a .^{21}$, no qual foi demonstrado que adultos vitimados pela fome durante a gestação no período da Segunda Guerra Mundial (Dutch Famine) apresentaram na vida adulta maior índice de massa corpórea e maior circunferência da cintura em relação aos indivíduos sem história de desnutrição.

A baixa estatura pode ser resultante da desnutrição na infância, sendo uma consequência da adaptação fisiológica à redução da oferta calórica no período de crescimento ${ }^{22-24}$. Assim, os estudos têm resultado em associação positiva da baixa estatura com a obesidade, o diabetes tipo 2, a hipertensão arterial e a dislipidemia ${ }^{7,16-20,25-28}$, que por sua vez constituem o conjunto de componentes da síndrome metabólica ${ }^{29,30}$. Entretanto, estudos de associação da baixa estatura com a síndrome metabólica são escassos na literatura. Dessa forma, o objetivo do estudo foi verificar a prevalência de síndrome metabólica $(\mathrm{SM})$ e sua relação com a baixa estatura em população adulta da região metropolitana de São Paulo (SP).

\section{Material e métodos}

\section{Casuística}

Trata-se de um estudo descritivo e analítico de corte transversal. A amostra foi composta por adultos com idade entre 20 e 64 anos, de ambos os sexos, selecionados aleatoriamente, por meio de sorteio de endereços a partir de uma listagem de todos os domicílios do município. Foram excluídos idosos, pois o encurtamento da coluna espinhal leva a uma perda de estatura, portadores de deficiência física, acamados e gestantes. $\mathrm{O}$ tamanho da amostra para regressão logística foi calculado por meio da fórmula de $\mathrm{Hsieh}^{31}$, utilizando dados do estudo piloto. O piloto teve como objetivo calcular a proporção $(\mathrm{p})$ de indivíduos com síndrome metabólica (variável dependente) e o coeficiente de correlação $\left(e^{2}\right)$ entre as variáveis independentes do modelo (baixa estatura, tabagismo, etilismo, sedentarismo, escolaridade, renda, sexo, idade e antecedentes familiares de doenças cardiovasculares, hipertensão e diabetes melito).

\section{Procedimentos e critérios de classificação}

Para dosagem da glicemia, triglicérides e colesterol total e frações, foi coletado sangue de jejum de, no mínimo, oito horas. Para classificação da SM adotou-se o critério preconizado pela International Diabetes Federation (IDF) ${ }^{30}$, ou seja, presença de obesidade abdominal, medida por meio da circunferência da cintura (>102 $\mathrm{cm}$ para homens e $>88 \mathrm{~cm}$ para mulheres) concomitante a alterações em dois ou mais outros componentes: glicemia de jejum $(\geq 100$ $\mathrm{mg} / \mathrm{dl}$ ), triglicérides, HDL-colesterol (<40 mg/ dl para homens e $<50 \mathrm{mg} / \mathrm{dl}$ para mulheres) e pressão arterial (sistólica $\geq 130 \mathrm{mmHg}$ e/ou diastólica $\geq 85 \mathrm{mmHg}$ ).

A aferição da circunferência da cintura foi obtida com fita métrica inelástica em centímetros no ponto médio entre a crista ilíaca e a face externa da última costela. A medida da pressão arterial foi obtida através da média de duas aferições, na posição sentada, após repouso e intervalo entre as aferições de cinco minutos.

A baixa estatura foi classificada por valores abaixo do percentil 5 (mulheres: $154 \mathrm{~cm}$; e homens: $165 \mathrm{~cm})^{32}$. 
O tabagismo foi classificado em duas categorias: fumante e não fumante.

O etilismo foi definido por meio do questionário $\mathrm{CAGE}^{33}$.

Para classificação do nível de atividade física (AF), utilizou-se o critério preconizado pela Organização Mundial da Saúde ${ }^{34}$. O cálculo baseiase na proporção entre o gasto energético total e a taxa de metabolismo basal do indivíduo em 24 horas.

\section{Análise estatística}

As associações entre estatura e SM foram feitas através de regressão logística multivariada, considerando como variáveis independentes idade, renda, nível de atividade física, tabagismo, etilismo e antecedentes familiares de doenças coronarianas, hipertensão arterial, diabetes.

A associação entre a SM e a baixa estatura foi avaliada por meio do cálculo de odds ratio (OR), controlando-se possíveis variáveis de confusão e investigando interações multiplicativas (modificação do efeito). Primeiramente, construiu-se o modelo univariado com a variável resposta (SM) e cada uma das variáveis independentes. Em seguida, as variáveis passíveis de entrada no modelo $(\mathrm{p}<0,05)$ foram sucessivamente acrescentadas, testando-se a cada passo a presença de confusão ou interação. Em geral, apenas mudanças na magnitude da OR superiores a $10 \%$ foram consideradas indicativas de confusão.

Foram considerados estatisticamente significantes resultados que apresentaram nível de significância menor que 5\% $(a=0,05)$ em intervalo de confiança de $95 \%$.

Toda a análise estatística foi realizada pelo SPSS Windows.

O projeto para este estudo teve aprovação do Comitê de Ética em Pesquisa da Faculdade de Saúde Pública da Universidade de São Paulo. Todo participante recebeu informações referentes à pesquisa e, após aceite, assinou o Termo de Consentimento Livre e Esclarecido, anterior a qualquer intervenção. Após recebimento dos exames, os indivíduos que apresentaram alterações nos dados bioquímicos investigados na pesquisa foram encaminhados para tratamento na própria Unidade Básica de Saúde.

\section{Resultados}

Na convocação, compareceram 214 indivíduos (74,6\%) do sexo feminino e 73 indivíduos $(25,4 \%)$ do sexo masculino. A população foi predominantemente de baixa renda e com baixo nível de escolaridade, sendo a renda per capita menor que R\$ 300,00 em 48,8\% da amostra e em 63,8\% até oito anos de escolaridade. Em relação aos aspectos demográficos, a amostra foi composta por um grande número de indivíduos migrantes da região Nordeste, perfazendo $35,9 \%$ da população estudada.

Em relação aos hábitos comportamentais, $22,6 \%$ eram tabagistas e 5,2\% etilistas. A proporção de sedentários foi alta na população, perfazendo $58,2 \%$ da amostra.

A Tabela 1 demonstra a prevalência padronizada segundo sexo e idade de síndrome metabólica e baixa estatura. Observa-se que $34,0 \%$ da população apresentaram SM e 29,0\% baixa estatura.

A Tabela 2 mostra a associação entre a SM e a baixa estatura, controladas pelas variáveis sexo, idade e características do bairro. Observa-se que a associação entre SM e baixa estatura mantémse significativa, sendo elas, portanto, independentes em relação a essas variáveis de controle.

A Tabela 3 mostra o modelo ajustado pelas variáveis sociodemográficas, comportamentais e antecedentes familiares de doenças coronarianas,

Tabela 1. Prevalência bruta $(\mathrm{PB})$ e prevalência padronizada (PP) da síndrome metabólica (SM) e baixa estatura - área metropolitana de São Paulo, 2006.

\begin{tabular}{lcrcc}
\hline & n & Casos & PB & PP* $^{*}$ \\
\hline SM & 287 & 105 & 36,6 & 34,0 \\
Baixa estatura & 287 & 93 & 32,4 & 29,0 \\
\hline
\end{tabular}

* Padronizada por sexo e idade.

Tabela 2. Associação entre síndrome metabólica e baixa estatura - área metropolitana de São Paulo, 2006.

\begin{tabular}{lcc}
\hline \multicolumn{1}{c}{ Modelos } & OR & IC (95\%) \\
\hline Baixa estatura & 1,308 & $1,298-1,319$ \\
Baixa estatura, sexo $^{*}$ & 1,240 & $1,230-1,250$ \\
Baixa estatura, idade $^{*}$ & 1,565 & $1,530-1,602$ \\
Modelo saturado $^{* *}$ & 1,247 & $1,118-1,257$ \\
\hline
\end{tabular}

$\mathrm{p}<0,001$.

"Inclui interação baixa estatura e idade; ${ }^{* *}$ Modelo ajustado por sexo e idade. 
hipertensão arterial e diabetes melito. Verifica-se que a associação entre SM e baixa estatura se mantém significativa em todos os modelos ajustados.

\section{Discussão}

O presente trabalho tem as limitações dos estudos transversais e, portanto, apresenta e discute associações entre eventos, sugerindo hipóteses que poderão ser mais bem exploradas em estudos de herdabilidade, entre outros. Sabe-se que SM se estabelece ao longo de muitos anos, e o que se pretendeu foi verificar associações entre essa morbidade e indicadores biológicos, socioeconômicos e comportamentais em um conjunto de indivíduos.

Pode-se presumir que a síndrome metabólica seja resultante de um longo processo que pode ter se iniciado nos primeiros anos de vida, até mesmo durante a gestaçãa ${ }^{10-12,35}$, com consequente prejuízo no crescimento estatural e as decorrentes adaptações metabólicas adversas da pobreza. A dimensão biológica e social da obesidade e da baixa estatura tem sido demonstrada em diversos estudos realizados em populações e $^{36-38}$. Assim, pode-se pressupor que a etiologia da SM é complexa, sendo determinada por fatores biológicos e socioambientais, como dieta, reações fisiológicas ao estresse, condições de vida (renda, educação, ocupação) etc.

Tabela 3. Associação entre síndrome metabólica e baixa estatura, controladas pelas variáveis sociodemográficas, comportamentais e antecedentes familiares de morbidades - área metropolitana de São Paulo, 2006.

\begin{tabular}{lrc}
\hline \multicolumn{1}{c}{ Modelos } & OR & IC (95\%) \\
\hline Baixa estatura, escolaridade & 1,257 & $1,224-1,294$ \\
Baixa estatura, renda per capita & 1,303 & $1,292-1,313$ \\
Baixa estatura, etilismo & 1,369 & $1,358-1,380$ \\
Baixa estatura, tabagismo & 1,313 & $1,303-1,323$ \\
Baixa estatura, atividade física & 1,279 & $1,269-1,289$ \\
Baixa estatura, HAS na família & 1,234 & $1,224-1,244$ \\
Baixa estatura, DC na família & 1,322 & $1,311-1,333$ \\
Baixa estatura, DM na família & 1,303 & $1,293-1,314$ \\
Modelo multivariado 1 * & 1,262 & $1,251-1,273$ \\
Modelo multivariado 2 ** & 1,166 & $1,156-1,177$
\end{tabular}

$\mathrm{p}<0,001$.

"Inclui baixa estatura, escolaridade, renda, etilismo, tabagismo, atividade física; ${ }^{* *}$ Inclui baixa estatura, antecedentes familiares de HAS (hipertensão arterial sistêmica), DC (doença coronariana) e DM (diabetes melito).
A correlação intrafamiliar positiva de estado nutricional de pais e filhos que compartilham tanto informações genéticas quanto condições socioeconômicas e ambientais também tem sido apontada por diversos pesquisadores. Martins et al. ${ }^{20}$, em estudo sobre a distribuição intrafamiliar de estados nutricionais, observaram relação significativa entre a baixa estatura de crianças e a de seus irmãos adolescentes e pais. A associação entre filho-mãe foi mais forte do que a encontrada entre filho-pai. A baixa estatura feminina foi associada à obesidade, permitindo a hipótese de que desnutrição pregressa e o excesso de peso, nessas mulheres, possam ser resultantes das adaptações metabólicas apontadas por Barker ${ }^{11,12}$. No entanto, em situações de pobreza o processo desnutrição-obesidade pode perpetuar-se das coortes mais velhas para as mais novas.

Guimarães et al. ${ }^{39}$ verificaram que os efeitos das variáveis sociais e daquelas marcadoras do potencial genético (por exemplo, a estatura dos pais) são condicionados pelo nível de desenvolvimento econômico e social da população estudada. Assim, poder-se-ia considerar que quanto mais alto o nível socioeconômico de populações, maior o potencial preditor da estatura dos pais em relação à estatura dos filhos. Portanto, em subgrupos populacionais de baixa renda e de baixa escolaridade, principais marcadores de situações precárias de sobrevivência, pode-se presumir que a baixa estatura seja determinada por fatores biológicos e sociais que podem ter se reproduzido através de gerações ${ }^{5,24}$. A baixa condição socioeconômica associa-se, ainda, a maiores taxas de mortalidade por doenças cardiovasculares e a outros fatores de risco como tabagismo, hipertensão, diabetes, sedentarismo e sobrepeso ${ }^{1,40}$.

A possível consequência de fatores adversos ao crescimento é a adaptação fisiológica à redução da oferta calórica pelo organismo. Nessas condições, o indivíduo reduz o gasto energético, acelerando deposição de gordura corporal, desencadeando a obesidade e comorbidades no decorrer da vida ${ }^{10-12}$. Em alguns estudos, o menor gasto energético foi demonstrado em $\mathrm{mu}$ lheres, o que poderia explicar o aumento, em particular, da obesidade no sexo feminino $0^{22,23,27}$.

Resultados de associação entre SM e baixa estatura são escassos na literatura. No presente estudo, a baixa estatura demonstrou associação positiva com a SM, o que corrobora a hipótese da SM como possível resultante da desnutrição na infância. A associação se manteve mesmo após ajuste por renda, escolaridade, etilismo, tabagismo, antecedentes familiares de DM, DC e HAS. 
Uma limitação do estudo foi o desequilíbrio na amostra final em relação aos sexos. Obtevese pequena amostra do sexo masculino em razão de recusas à avaliação nas Unidades Básicas de Saúde, por motivo de trabalho ou por desinteresse em comparecer à UBS (a população do sexo feminino é predominante em toda a rede básica do local de estudo). Por isso, as análises realizadas não abordaram diferenciais por gênero.

A baixa estatura como indicador de privações nutricionais na infância pode ser fator para morbidades na vida adulta, como tem sido descrito por vários autores e demonstrado neste estudo. Considerando que desnutrição em crianças é um problema de saúde pública em países em desenvolvimento, esses achados poderiam ter implicações importantes para a prevenção de morbidades associadas à SM.

\section{Colaboradores}

EC Silva, IS Martins e EAC Araújo participaram da escolha da população, do modelo proposto e da estatística aplicada. EC Silva redigiu a versão final do texto, revisada por IS Martins.

\section{Referências}

1. World Health Organization. Food and Agriculture Organization (WHO/FAO). Diet, nutrition and prevention of chronic disease. WHO Technical Report Series. Geneva: WHO; 2003.

2. Perestrelo JP, Martins IS. Modernização rural: transformações econômicas e suas implicações demográficas, epidemiológicas e sociais. Saúde Socied 2003; 12:38-55.

3. World Health Organization. The Asia-Pacific perspective: redefining obesity treatment. WHO Western Pacific Region. Geneva: WHO; 2000.

4. Popkin BM, Doak C. The obesity epidemic is a worldwide phenomenon. Nut Rev 1998; 56:95-103.

5. Batista Filho M, Rissin A. A transição nutricional no Brasil: tendências regionais e temporais. Cad Saude Pub 2003; 19(1):181-191.

6. Monteiro CA, Benicio MHDA, Conde WL, Popkin BM. Shifting obesity trends in Brazil. Eur J Clin Nutr 2000; 54:342-346.

7. Prentice AM, Moore SE. Early programming of adult disease in resource poor countries. Arch Dis Child 2005; 90:429-432.

8. Florêncio TT, Ferreira HS, França AP, Cavalcante JC, Sawaya AL. Obesity and undernutrition in a very low income population in the city of Maceió, Northeastern Brazil. Brit J Nutr 2001; 86:277-284.

9. Popkin BM. The nutrition transition and its health implication in low-income countries. Pub Health Nutr 1998; 1(1):5-21. 
10. Waterland RA, Garza C. Potential mechanisms of metabolic imprinting that lead to chronic disease. Am J Clin Nutr 1999; 69:179-197.

11. Barker DJP. Maternal nutrition, fetal nutrition, and disease in later life. Nutrition 1997; 13(9):807-813.

12. Barker DJP, Gluckman PD, Godfrey KM, Harding JE, Owens JA, Robinson JS. Fetal nutrition and cardiovascular disease in adult life. Lancet 1993; 341:938-941.

13. Parker DR, Lapane KL, Lasater TM, Carleton RA. Short stature and cardiovascular disease among men and women from two Southeastern New England communities. Int J Epidemiol 1998; 27:970-975.

14. Wamala SP, Mittleman MA, Horsten M, SchenckGustafsson K, Orth-Gomér K. Short stature and prognosis of coronary heart disease in women. $J$ Internal Med 1999; 245:557-563.

15. Velasquez-Meléndez G, Martins IS, Cervato AM, Marucci FN, Coelho LT. Relationship between stature, overweight and central obesity in the adult population in São Paulo, Brazil. Int J Obes 1999; 23:639-644.

16. Sichieri R, Siqueira KS, Moura AS. Obesity and abdominal fatness associated with undernutrition early in life in a survey in Rio de Janeiro. Int $J$ Obes Relat Metab Disord 2000; 24:614-618.

17. Sichieri R, Siqueira KS, Pereira RA, Ascherio A. Short stature and hypertension in the city of Rio de Janeiro, Brazil. Pub Health Nutr 1999; 3:77-82.

18. Sawaya AL, Martins P, Hoffman D, Roberts S. The link between childhood undernutrition and risk of chronic disease in adulthood: a case study of Brazil. Nutrition 2003; 61(5):168-175.

19. Sawaya AL, Roberts S. Stunting and future risk of obesity: principal physiological mechanisms. Cad Saude Pub 2003; 19(Supl.1):21-28.

20. Martins IS, Marinho SP, Oliveira DC, Araújo EAC. Pobreza, desnutrição e obesidade: inter-relação de estados nutricionais de indivíduos de uma mesma família. Cien Saude Colet 2007; 12(6):1553-1565.

21. Ravelli GP, Stein Z, Susser M. Obesity in young men after famine exposure in utero and early infancy. N E J Med 1976; 295:349-353.

22. Hoffman DJ, Sawaya AL, Verreschi I, Tucker KL, Roberts SB. Why are nutritionally stunted children at increased risk of obesity? Studies of metabolic rate and fat oxidation in shantytown children from Sao Paulo, Brazil. Am J Clin Nutr 2000; 72:702-707.

23. Hoffman DJ, Sawaya AL, Coward WA, Wright A, Martins PA, Nascimento C, Tucker KL, Roberts SB. Energy expenditure of stunted and nonstunted boys and girls living in the shantytowns of São Paulo, Brazil. Am J Clin Nutr 2000; 72:1025-1031.

24. Monteiro CA, Conde WL. Tendência secular da obesidade segundo estratos sociais: Nordeste e Sudeste do Brasil, 1975-1989-1997. Arq Bras Endocrinol Metabol 1999; 43:186-194.

25. Langenberg C, Hardy R, Breeze E, Kuh D, Wadsworth MEJ. Influence of short stature on the change in pulse pressure, systolic and diastolic blood pressure from age 36 to 53 age: an analysis using multilevel models. Int J Epidemiol 2005; 34(4):905-913.

26. Florêncio TT, Ferreira HS, Cavalcante JC, Sawaya AL. Short stature, obesity and arterial hypertension in a very low income population in Northeastern Brazil. Metab Cardiov Dis 2004; 14(1):26-33.
27. Sawaya AL, Grillo LP, Verreschi I, Silva AC, Roberts SB. Mild stunting is associated with higher susceptibility to the effects of high fat diets: studies in a shantytown population in Sao Paulo, Brazil. J Nutr 1998; 128:415-420.

28. Sawaya AL, Dallal G, Solymos GM, Sousa MH, Ventura ML, Roberts SB, Sigulem DM. Obesity and malnutrition in a shantytown population in the city of Sao Paulo, Brazil. Obes Res 1995; 3:107-115.

29. Zimme P, Magliano D, Matsuzawa Y, Albert G, Shaw J. The metabolic syndrome: a global public health problem and a new definition. I Atherosclerose Thromb 2005; 12(6):295-300.

30. International Diabetes Federation. The IDF consensus worldwide definition of the metabolic syndrome [periódico na Internet] 2005; [acessado 2007 jun 12], 7p. Disponível em: http://www.pitt.edu/ $\sim$ super1/Metabolic/IDF1.pdf

31. Hsieh FY. Sample size tables for logistic regression. Statist Med 1989; 8:795-802.

32. Ogden CL, Kuczmarski RJ, Flegal KM. Centers for Disease Control and Prevention 2000 growth charts for the United States: improvements to the 1977 National Center for Health Statistics Version. Pediatrics 2002; 109:45-60.

33. Masur J, Capriglione MJ, Monteiro MG, Jorge MR. Deteç̧ão precoce do alcoolismo em clínica médica através do questionário CAGE: utilidade e limitação. J Bras Psiquiatr 1985; 34(1):31-34.

34. FAO/OMS/ONU. Necesidades de energia $y$ de proteínas. Ginebra: OMS; 1985.

35. Ijzerman RG, Stehouwer CD, Boomsma DI. Intrauterine environmental and genetic influences on the association between birthweigth and cardiovascular risk in twins as a means of testing the fetal origin hypotheses. Pediatr Perinat Epidemiol 1990; 19(Supl.1):10-14.

36. Engstrom EM, Anjos LA. Déficit estatural nas crianças brasileiras: relação com condições socioambientais e estado nutricional materno. Cad Saude Publica 1999; 15:559-567.

37. Floud R, Watcher K, Gregory A. A long term trend in nutritional status. In: Floud R, Watcher K, Gregory A, editors. Height, health and history: nutritional status in United Kingdom 1750-1980. Cambridge: Cambridge University Press; 1990. p. 134-195.

38. Tarakan CT, Suchindran CM. Determinants of child malnutrition: an intervention model for Botswana. Nutrition Research 1990; 19(6):843-860.

39. Guimarães LV, Latorre MRDO, Barros MBA. Fatores de risco para a ocorrência de déficit estatural em pré-escolares. Cad Saude Publica 1999; 15(3):605-615.

40. World Health Organization. The World Health Report. Geneva: WHO; 1998. (WHO Technical Report Series).

Artigo apresentado em 26/12/2007

Aprovado em 04/06/2008

Versão final apresentada em 04/07/2008 